Z. klin. Chem. u. klin. Biochem.

9. Jg., S. 164-174, März 1971

\title{
Neuere Aspekte der Kininforschung
}

\author{
Von Rosmarie Vogel, E. Werde und Gertrud Zickgraf-Rüded'
}

\section{Potenzierung und Blockierung der biologischen Kininwirkung}

Von Rosmarie Vogei und Gertrud Zickgraf-Rüdel

Institut fïr Klinische Chemie und Klinische Biochemie der Universität München

(Direktor: Prof. Dr. Dr. E. Werle)

Teil 2: Blockierung

(Eingegangen am 19. Februar/28. Mai 1970)

\begin{abstract}
Der zweite Teil der Übersicht über pharmakologische Beeinflussung der Kininwirkung behandelt deren Blockierung durch Kininanaloga, Analgetica und Antipyretica mit peripherer und zentraler Wirkung, entzündungshemmende Steroide, Substanzen mit Wirkung auf das periphere Nervensystem und das cardiovasculäre System, Histamin- bzw. Serotoninantagonisten ${ }^{1}$ ). In die Diskussion sind speziell hinsichtlich des Wirkungsmechanismus nur Arbeiten einbezogen, die die direkte Beeinflussung der Kininwirkung beinhalten. Keine der getesteten Substanzen hemmt die Kininliberierung. Der Hemm-Mechanismus, Unterschiede in den Ergebnissen bei Verwendung verschiedener Spezies und die Übertragbarkeit der Experimente auf patho-physiologișche Vorgänge werden diskutiert. Es ergeben sich daraus Hinweise auf die Art der Kinin-Rezeptoren und den Wirkungsmechanismus der Kinine selbst.
\end{abstract}

The second part of the review of the pharmacological influence on kinins deals with the blockage of their action by kinin analogues, analgesics and antipyretics with peripheral and central action, anti-inflammatory steroids, substances with activity in the peripheral nervous system and cardiovascular system, and histamine and serotonin antagonists. The discussion of the mechanism of action is limited to work that deals with the direct effects of kinins. None of the tested substances inhibits the liberation of kinins. The mechanism of inhibition, differences between species, and the relevance of these experiments to the interpretation of patho-physiological processes are discussed. Information is thus obtained on the nature of the kinin receptors and the action mechanism of the kinins themselves.

Die Suche nach Kinin-Antagonisten verfolgt zwei Ziele: blockierende Substanzen unter den Analoga der Kinine zu finden oder eine direkte Antikininwirkung bei Substanzen nachzuweisen, deren bekannte pharmakologische Effekte mit den pharmakologischen Wirkungen der Kinine (s. Tab. 1 in Teil $1^{2}$ )) in Verbindung stehen.

\section{Kinin-Analoga}

Die Suche nach Bradykinin-Analoga mit AntikininEffekt wurde angeregt durch die Auffindung von Oxytocin- und MSH (Melanophoren-stimulierendes Hormon)-Analoga mit Antioxytocin- bzw. Anti-MSHWirkung. Die Merrifield-Methode gestattet eine rasche Synthese von vielfältigen Derivaten des Moleküls, deren Auswahl die Studien über die Bedeutung der einzelnen Positionen im Kininmolekül für die Bindung an den Rezeptor bestimmten.

Die Bedeutung der einzelnen Aminosäurepositionen für die biologische Aktivität (aufgrund der pharmakologischen Eigenschaften der bisher untersuchten

1) Die Nomenklatur der referierten Substanzen wurde nach $H$. IPPEN, Index Pharmacorum 1968 vereinheitlicht. Soweit die Kinindosierung nicht angegeben ist, bält sie sich in der Größenordnung der in Tabelle 1 des Teil $1^{2}$ ) angegebenen Testdosis.

$\left.{ }^{2}\right)$ diese Z. 8, 177 (1970).
Bradykinin-Analoga) entspricht abnehmend folgender Reihe: $\mathrm{Pro}^{7}=\mathrm{Phe}^{8}>\mathrm{Arg}^{1}>\mathrm{Arg}^{9}>\mathrm{Phe}^{5}>\mathrm{Gly}^{4}>$ $\mathrm{Pro}^{2}>\mathrm{Pro}^{3}>\mathrm{Ser}^{6}(1)$

Die Zusammenhänge zwischen der Struktur vieler Analoga und ihrer biologischen Aktivität (1,2 und Übersicht in 3) sind ziemlich genau bekannt. Das Carboxylende der Kette muß für die Bindung an den physiologischen Rezeptor unverändert sein, ebenso die Länge der Seitenketten in Position 1 und 9 (4). Die Bedeutung der Position 9 ist bereits damit unterstrichen, daß die natürliche enzymatische Inaktivierung des Bradykinins durch Abspaltung des C-terminalen Arginins erfolgt (5).

Ziel der Synthese von Antagonisten ist die Änderung im Molekül, die das Peptid stabilisiert gegenüber dem Abbau durch Carboxypeptidasen oder Chymotrypsin, d. h. Änderung in Position 9 bzw. 5 oder 8. Daher ist außer weiteren Veränderungen im Molekül der größte Teil der in Tabelle 1 und 2 aufgeführten Analoga in Position 5 und 8 substituiert; stärkster bisher bekannter Antagonist ist 5,8-Di-O-methyltyrosin-Bradykinin (Substanz 4) (6). Verlängerung der Kette am C-terminalen Ende führt zu starker Verminderung der $\mathrm{Ak}$ tivität und bei einem der Analoga mit zusätzlicher Substitution in Position 6 zur Hemmwirkung (Sub$\operatorname{stanz} 3)$. 
Tab. 1

Blockierung der Bradyki,inwirkung in vitro (Kontraktion des Rattenuterus) durch Bradykininanaloga (nach STEWART (4, 6-8)) Vorinkubation mit dem Analogon für 4 Min., Bradykininzugabe $(0,001-0,003 \mu \mathrm{g} / \mathrm{ml})$ ohne Zwischenspülung

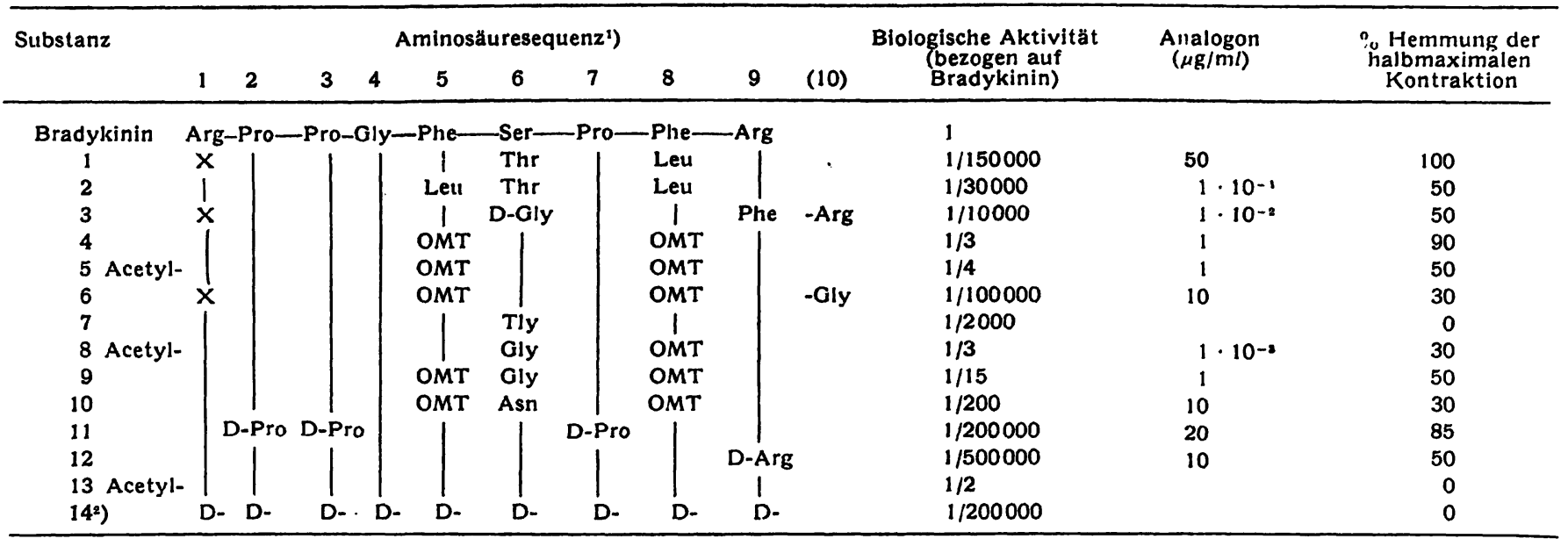

1) $\mathrm{OMT}=$ Tryrosinmethyläther, Tly $=6$-Tosyllysin, $\mathrm{X}=$ Des-.

2)All-D-Bradykinin

Tab. 2

Blockierung der Bradykininwirkung in vivo (Permeabilitätserhöhung . beim Meerschweinchen) durch Bradykininanaloga (nach STEWART $(4,6-8)$ ). Intradermale Injektion eines Bradykinin $(0,01 \mu \mathrm{g})$-Analogon-Gemisches

\begin{tabular}{|c|c|c|c|}
\hline $\begin{array}{c}\text { Substanz } \\
\text { (vgl. Tab. 1) }\end{array}$ & $\begin{array}{l}\text { Biologische Ak- } \\
\text { tivität, bezogen } \\
\text { auf Bradykinin }\end{array}$ & $\begin{array}{c}\text { Analogen } \\
\text { ( } \mu \mathrm{g} / \text { Injektion } \\
\text { im Gemisch) }\end{array}$ & $\begin{array}{l}\because \text { Hemmung } \\
\text { der Permeabili- } \\
\text { tätserhöhung }\end{array}$ \\
\hline Bradykinin & 1 & & \\
\hline $\begin{array}{r}7 \\
8 \\
11 \\
13 \\
14\end{array}$ & $\begin{array}{l}1 / 100 \\
1 / 10 \\
1 / 300 \\
1 / 5 \\
1 / 200\end{array}$ & $\begin{array}{l}1 \cdot 10^{-8} \\
1 \cdot 10^{-4} \\
1 \cdot 10^{-4} \\
1 \cdot 10^{-5} \\
1 \cdot 10^{-1}\end{array}$ & $\begin{array}{l}25 \\
50 \\
28 \\
35 \\
35\end{array}$ \\
\hline
\end{tabular}

Die Bedeutung der Positionen 5 und 8 (beides Phe) ist auf den aromatischen Ring zurückzuführen, da offensichtlich die $\pi$-Elektronen mit denen des Rezeptors in Wechselwirkung treten (4). So führt die Substitution von Phenylalanin durch Tyrosin-methyläther zu stark verminderter pharmakologischer Aktivität bzw. antagonistischer Wirkung (s. Tab. 1, Analoga 4, 5 und 6). Bei Peptiden führt im allgemeinen die Einführung von Methylgruppen, d. h. ganz allgemein die Vergrößerung von „Positionen“, die normal mit Serin besetzt sind, zu Antimetaboliten (im Fall von Bradykinin: Analoga 1 und 2).

Totale oder partielle optische Inversion führten bei einigen anderen Peptiden zur Auffindung von Antimetaboliten; bei all-D-Bradykinin (Substanz 14) ist die pharmakologische Wirkung am Rattenuterus auf $1 / 200000$ gesenkt, eine Antikininwirkung in vitro nicht $z u$ beobachten (7). Die pharmakologische Wirkung des D-Pro 2,3,7-Bradykinins (Substanz 11) ist um denselben Faktor am Rattenuterus verringert; es ist ein schwacher Antagonist in vitro. Des-Arg-D-Pro 2,3.7Bradykinin, das Analogon von Substanz 11, hat dagegen keine Hemmwirkung. Ausgeprägten Antikinin-Effekt hat D-Arg ${ }^{9}$-Bradykinin (Substanz 12), was wieder die Bedeutung des Carboxylendes unterstreicht.

Einige Analoga differieren sehr in ihrer antagonistischen Wirkung in vitro (Rattenuterus) und in vivo (Kapillarpermeabilität, Meerschweinchen) $(4,8)$ (s. Tab. 1 und 2), wobei die Analoga mit acetylierter $\alpha$-Aminogruppe
(Substanz 13) und die mit optisch isomeren Aminosäuren substituierten (Substanzen 11 und 14) hervorzuheben sind. Substanz 14 zeigt jedoch keine Hemmwirkung am Blutdruck der Katze (9).

Das O-Acetyl-threonin-methylester-derivat von Substanz 2 bewirkt interessanter Weise 100proz. spezifische irreversible Hemmung von Bradykinin am Uterus von galaktosämischen Ratten (6). Experimentell konnte Woolley den Nachweis erbringen, daß der SerotoninRezeptor ein Galaktolipid ist, der z. B. im Gewebe von Rattenmägen durch Neuraminidase oder EDTA zerstört wird, während der Bradykinin-Rezeptor erhalten bleibt; doch ist offensichtlich Galaktose an der $\mathrm{Zu}$ sammensetzung des Bradykinin-Rezeptors beteiligt (7). So zeigen diese Untersuchungen einzelne Erfolge; im allgemeinen gesehen, ist aber der Kinin-Antagonist unter den Analoga noch nicht gefunden, denn die Autoren betonen immer die Inkonstanz des Effektes, d. h. große Streubreite bei verschiedenen Versuchstieren (s: S. 171). Der Hemmeffekt ist auch immer in höherer Konzentration des Analogons begleitet von einer Kinineigenwirkung.

\section{Hemmwirkung von Peptiden}

Von verschiedenen, von YAMAMURA untersuchten niederen Peptiden hat sich nur $\mathrm{N}^{\varepsilon}$-Formyl-L-lysin als Blocker von Bradykinin erwiesen. Die Prüfung des antagonistischen Effektes erfolgte nur bei der Kinininduzierten Kapillarpermeabilitätserhöhung bei Meerschweinchen (10).

Analgetica, Antipyretica, Antineuralgica ohne Histamin- oder Serotonineffekt (Tab. 3a)

\section{Salicylsäure und-derivate}

Schachter, Collier und Shorley fanden 1959, daß i. v. applizierte Acetylsalicylsäure beim Meerschwein- 
chen die Kinin-induzierte Bronchokonstriktion blokkiert $(11,12)$. Es zcigte sich aber schon an dieser ersten als Blocker bekanntgewordenen Substanz, daß bei Verwendung anderer Tierspezies oder ${ }^{\circ}$ Test-Organe der Hemmeffekt ausbleiben kann (13). Dagegen wird die bronchokonstriktorische Wirkung von Bradykinin und Kallidin und - soweit untersucht - ihrer Analoga blockiert $(13,14)$.

Diese ersten Untersuchungen wurden von verschiedenen Arbeitsgruppen bestätigt, die die antagonistische Wirkung der Salicylsäure und deren Derivate in vitro und in vivo studierten (Tab. 3a).

Aus der Menge der Befunde sei herausgehoben: Acetylsalicylsäure und Vertreter anderer Substanzklassen (s. weiter unten) reduzieren die Höhe des Blutdruckabfalls nach Bradykiningabe nicht, aber die Zeit des Wiederanstiegs des Blutdrucks wird verkürzt.

Die Bronchialwirkung von Histamin, Serotonin, Acetylcholin (14) und auch von Eledoisin (15) bleibt unbeeinflußt.

Es scheint, daß der Kinin-antagonistische Effekt auf die Rezeptoren der Bronchialmuskulatur beschränkt ist, da die Hemmung der Bronchokonstriktion auch nach Durchtrennuing der spinalen und autonomen Nervenfasern der Lunge bestehen bleibt (16). Möglich ist auch eine Blockierung der Verbindung Rezeptor-Kinin (17), oder die Hemmung ist keine echte im Sinne einer Substanz-Rezeptor-Reaktion (18).

\section{Derivate der Phenylessigsäıre}

Die Blockierung der Kininwirkung durch Ibufenac und Ibuprofen wurde nur am Blutdruck getestet; weitere Untersuchungen beziehen sich besonders auf die Beeinflussung verschiedener Ödemformen (s. Tab. 3a).

\section{Derivate der Anilinobenzoesäure ${ }^{3}$ ) (Tab. 3b)}

Der bestuntersuchte Vertreter dieser Gruppe mit bekannter antiphlogistischer bzw. antineuralgischer Wirkung ist die Flufenamsäure. Wenn auch die direkte Antikininwirkung nur bei einigen pharmakologischen Tests nachgewiesen ist, so ist sie in großen Statistiken indirekt erwiesen bei pathophysiologischen Zuständen, bei denen eine Beteiligung des Kininogenase-KininSystems angenommen wird.

Für die direkte Kininblockierung sprechen die Hemmung der durch Bradykinin ausgelösten Bronchokonstriktion beim Meerschweinchen (in vivo) durch mehrere Vertreter dieser Substanzgruppe $(17,35,43$, 44). Die Minimaldosis des Antagonisten wurde getestet durch Vergleich der Wirkung von i. v. appliziertem Bradykinin $(0,125$ und $1,25 \mu \mathrm{g} / \mathrm{kg}) 20$ und 10 Min. bzw. 30 Sek. nach der i. v. Applikation des zu testenden Antagonisten. Nach Collrer und Mitarbeitern (43) ist die Minimaldosis, i. v. appliziert, von Meclofenamsäure $0,06 \mathrm{mg} / \mathrm{kg}$ sehr niedrig im Ver-

3) Die Substanzen wurden als $\mathrm{Na}$-Salz appliziert. gleich zu anderen Substanzgruppen. Auch beim chronischen Asthmatiker wirkt Flufenamsäure hemmend auf den Bronchospasmus (31).

Die trisubstituierten Derivate der Anilinobenzoesäure scheinen nach WINDER und Mitarbeitern (34) stärker wirksam bei Blockade des UV-Erythems als die disubstituierten. Eines der untersuchten Derivate ist CI-583. Die Arbeitsgruppe GRYGLEWSKI hat sie in vergleichende Untersuchungen der Antibradykininwirkung disubstituierter Fenamsäuren einbezogen (19) und fand, daß CI-583 (2-[2.6-Dichlor-3-methyl-anilino]benzoesäure) kein spezifis cher Bradykinin-Antagonist ist (s. Tab. 3b).

GrYGLEwSKI und Mitarbeiter (19) fanden an isolierten Organen für Flufenamsäure Abfall der Kininblockierenden Wirkung in der Reihenfolge: Trachea, Meerschweinchen $>$ Duodenum, Ratte $>$ Ileum, Meerschweinchen $>$ Magen, Ratte $>$ Arterien, Kaninchen. Die Hemmung scheint nicht kompetitiv zu sein. Nach den von Rocha e SrLva als $\mathrm{Maß}$ für seine Substanzen angegebenen $\mathrm{pK}_{\mathrm{i}}$-Werten (45) ist Flufenamsäure $100 \mathrm{mal}$ wirksamer als die von Rocha e Silva gefundene wirksamste tricyclische Substanz (s. S. 167). Am isol. Meerschweinchenileum scheint ASD 30 (2[2.6-Dichlor-3-benzylthio-anilino]-benzoesäure ebenfalls ein nicht-kompetitiver Bradykinin-Antagonist zu sein (46). Bei Vorgabe von $\geqq 20 \mu \mathrm{Mol} / \mathrm{ml}$ für $20 \mathrm{Min}$. war die pharmakologische Wirkung von $0,1-25 \mu \mathrm{g}$ Bradykinin $/ \mathrm{m} l$ nicht nur auf $50 \%$ erniedrigt, sondern auch nach $1 \mathrm{Std}$. Spülung nicht mehr voll reproduzierbar. (Dosisabhängige Bradykininhemmung ab $3 \mu \mathrm{Mol} / \mathrm{ml}$.)

STARR und WeSt vergleichen die hemmende Wirkung beim experimentellen Pfotenödem mit der Blockierung der Bradykinin-induzierten Permeabilitätserhöhung (32). Während die Fenamate (i. p. $100 \mathrm{mg} / \mathrm{kg}$ ) eine ziemlich stark dämpfende Wirkung auf das thermische Rattenpfotenödem haben, sind sie bei Ödemen nach i. d. Applikation von Bradykinin nur wirksam, wenn sie i. p. appliziert werden, aber nicht bei lokaler Applikation. Bei i. p. Applikation fanden STARR und WEST auch eine Blockierung der Histamin- und Serotonininduzierten Permeabilitätserhöhung.

Die Hemmung der Bradykinin-induzierten Bronchokonstriktion wird nicht beeinflußt durch Ausschaltung von Gehirn und Rückenmark, durch bilaterale Adrenalektomie oder $\beta$-Rezeptoren-Blockade.

Ein antagonistischer Effekt der ganzen Substanzgruppe gegenüber der Kinin-induzierten Catecholaminfreisetzung wird nicht beobachtet (43).

Diese Untersuchungen tragen wesentlich zur Kenntnis des Wirkungsmechanismus dieser Substanzgruppe bei; sie wirkt nicht über Nervensystem, Catecholamine, aber auch nicht über die Hemmung der Kininliberierung: nach Davies und LOWE und Mitarbeitern (22) hemmt Flufenamsäure $(100 \mu \mathrm{g} / \mathrm{m} /)$ die Kininfreisetzung in einem Testansatz mit gereinigtem Kallikrein aus Meerschweinchenserum und Rinderkininogen nicht. 
Pyrazolonderivale (Tab. 3c)

Das hinsichtlich der Kinin-antagonistischen Wirkung bestuntersuchte Pharmakon ist Phenylbutazon. Es blockiert praktisch alle Kininwirkungen und es sind auch fast alle Befunde von verschiedenen Arbeitsgruppen bestätigt worden.

Bevorzugt bezieht sich die Hemmung von Bradykinin auf dessen Bronchialwirkung; Histamin, Serotonin, Acetylcholin und auch Eledoisin werden dagegen nicht blockiert, selbst in einer mehrfachen Bradykininantagonistischen Dosis (15, 52), wie auch (s. S. 171) Histamin-Antagonisten keine Blocker der Kinin-induzierten Bronchokonstriktion sind (54). Hier liegt eine eindeutige Spezifizierung in bezug auf die Rezeptoren vor.

Auch hinsichtlich der entzündungshemmenden Wirkung von Phenylbutazon ist nach Experimenten von VARgaftig und Bonta (Lit. s. Tab. 3c) sicher, daß sie teilweise auf dem Kinin-antagonistischen Effekt beruht. Durch Vergleich des Kiningehaltes in der Flüssigkeit aus verschiedenartig induzierten Ödemen (z. B. durch Carrageen, Serotonin oder Kaolin einerseits und Polyvinylpyrrolidon andererseits) konnten Bonta $(55,56)$ und VARgaftig und Mitarbeiter (57) zeigen, daß Phenylbutazon nur Ödemtypen hemmt, die von einer lokal erhöhten Kininfreisetzung begleitet sind (z. B. Carrageen-Ödem). Dazu fügt sich ein von STARR und WEST (32) nu r für Phenylbutazon erhobener Befund: Die Kapillarpermeabilitätserhöhung bei der Ratte durch intra-dermal appliziertes Bradykinin wird nicht nur blockiert durch i. p. appliziertes Phenylbutazon, sondern ebenso durch i. d. appliziertes $(1,0 \mu \mathrm{g}$ Bradykinin zu $50 \%$ durch $1,0 \mathrm{mg}$ Phenylbutazon). Allerdings tritt bei diesem Test auch eine Hemmung der Histamin- bzw. Serotoninwirkung auf.

Bei intraventrikulärer Injektion löst Bradykinin Vokalisation und andere Anzeichen von Schmerz beim Versuchstier aus, Wirkungen, die bei PhenylbutazonPrämedikation ausbleiben. GREEFF und RochA E Silva (58) deuten dies mit einer direkten Kinin-Rezeptorblockade im ZNS.

\section{Derivate anderer Heterocyclen (Tab. 3d)}

Experimente, die mit Indozol, Indomethazin, Benzindamin, ID-955 (1-3',4'-Methylendioxybenzoyl-2-methyl-5-methoxy-3-indolylessigsäure), ICI-54450 (2-4'Chlorphenyl-thiazolyl-4-essigsäure) und Chloroquin ausgeführt wurden, sollen nur tabellarisch zusammengefaßt werden, da die Ergebnisse noch sehr lückenhaft sind und daher nur ein Vergleich mit 'den anderen Gruppen gegeben werden kann (Tab. 3d).

\section{Entzindungsbemmende Steroide (Tab. 3e)}

ShImamoto stellte fest, daß Oestrogene in vivo KininAntagonisten sein können. Diese Untersuchungen gehen auf Beobachtungen von LuRIE (68) bzw. BoNTA und $\mathrm{DE}$ Vos (69) zurück.

Außer für den antagonistischen Effekt auf die Venokonstriktion (70) (Kritik s. Pyridinolcạrbamat,. S. 170) konnten Shimamoro und Mitarbeiter (71) den Nachweis für die Hemmung der Kapillarpermeabilitätssteigerung durch Bradykinin erbringen. Dieser Effekt ist nur bei bestimmten Kinin-Oestrogen-Mischverhältnissen signifikant, bei Verschiebungen der Dosen tritt er dagegen nicht auf. Eine signifikante Hemmung der Histaminwirkung wurde von Shimamoro nicht beobachtet.

Bonta (56) findet dagegen, daß das durch Histamin bzw. Serotonin ausgelöste Rattenpfotenödem durch Oestriolsuccinat signifikant gehemmt wird, betont allerdings, daß die Substanz selbst die Gefäßpermeabilität erniedrigt.

Crine und Melmon (72) berichten, daß Glaskontaktaktivierung und Kininliberierung durch Kallikrein in vitro von Cortisol ( 2 bis $20 \mu \mathrm{M}$ ) blockiert werden kann. Aus Blut von Prednisolon-behandelten Patienten kann kein Kinin entwickelt werden.

Alle Befunde, die mit entzündungshemmenden Steroiden erhoben wurden, erscheinen EISEN, GREENBAUM und LEwrs (73), die unabhängig voneinander arbeiteten und zu demselben Ergebnis gelangten, fragwürdig, da Hydrocortison, Prednisolon und Dexamethason verschiedene Arten der Kinin-Liberierung in vitro nicht hemmen. Die Autoren ziehen daraus den Schluß, daß Corticoide nicht über die Blockierung des Kinin-Systems entzündungshemmend wirken. Beim Vergleich mit anderen Substanzgruppen fällt jedoch auf, daß prinzipiell von Kininblockern die Kininliberierung nicht gehemmt wird, sondern sich immer nur eine direkte Kinin- bzw. Kinin-Rezeptor-Blockade nachweisen läßt.

Da die Experimente hinsichtlich der direkten KininBlockierung noch sehr einseitig sind (s. Tab. 3e) und viele negative Ergebnisse gebracht haben, ist die Frage nach der antagonistischen Wirkung von Steroiden nur durch Erweiterung der Tests in vitro und in vivo $z u$ klären. Man ist geneigt, sich der Meinung von BoNrA (56) anzuschließen, daß die Dämpfung der Gefäßpermeabilität bei dieser Substanzgruppe durch direkte Wirkung auf die Gefäßwand oder auf umgebende Gewebsbestandteile ausgelöst wird und mit einer spezifischen Blockade der Kinine nichts zu tun hat.

\section{Zentrale Analgetica (Tab. 3f)}

In die Untersuchungen wurden auch kondensierte Heterocyclen einbezogen, eine Substanzgruppe mit bekannter dämpfender Wirkung auf das ZNS.

Codein und Morphin vermögen die durch KininApplikation ausgelöste Schmerzreaktion, und zwar sowohl den visceralen Schmerz als auch die Feldpotentiale des Cortex-Assoziationsfeldes zu blockieren (Lit. s. Tab. 3f). Nach Lewrs und ReIT (78) und TreNDELENBURG (79) blockiert Morphin die Ganglienstimulierung durch Kinine. Die Substanzen zeigen in vitro am isolierten Meerschweinchenileum Bradykinin-antagonistischen Effekt (Lit. s. Tab. $3 \mathrm{f}$ ).

Konzert (87) fand am isolierten Kaninchendarm als Bradykinin-Antagonisten in Dosen von $\geqq 10 \mu \mathrm{g} / \mathrm{m} l$ 


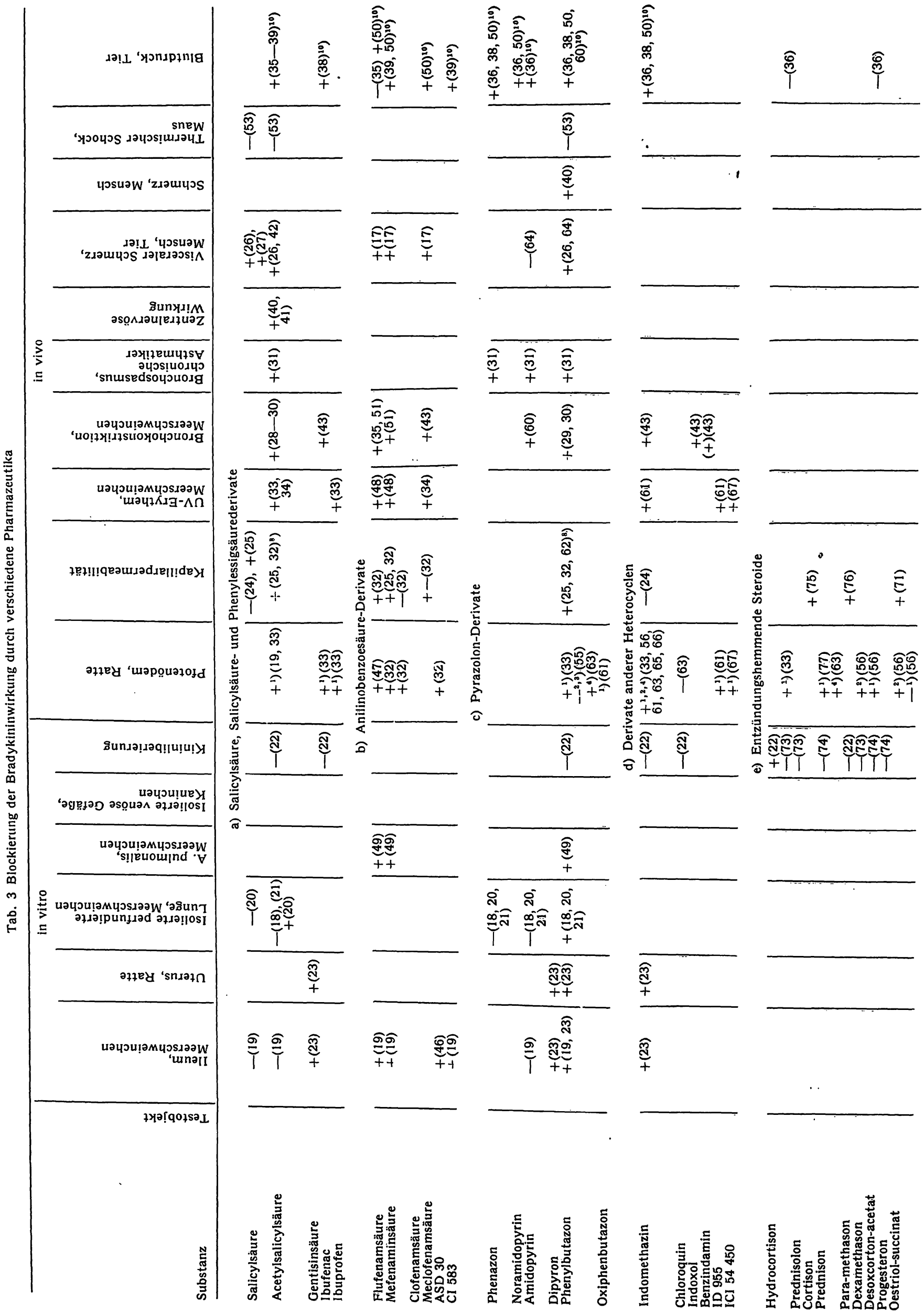

Z. klin. Chem. u. klin. Biochem. / 9. Jahrg. 1971 / Heft 2 


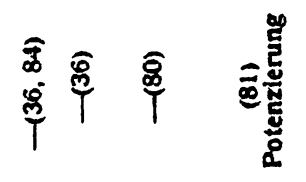
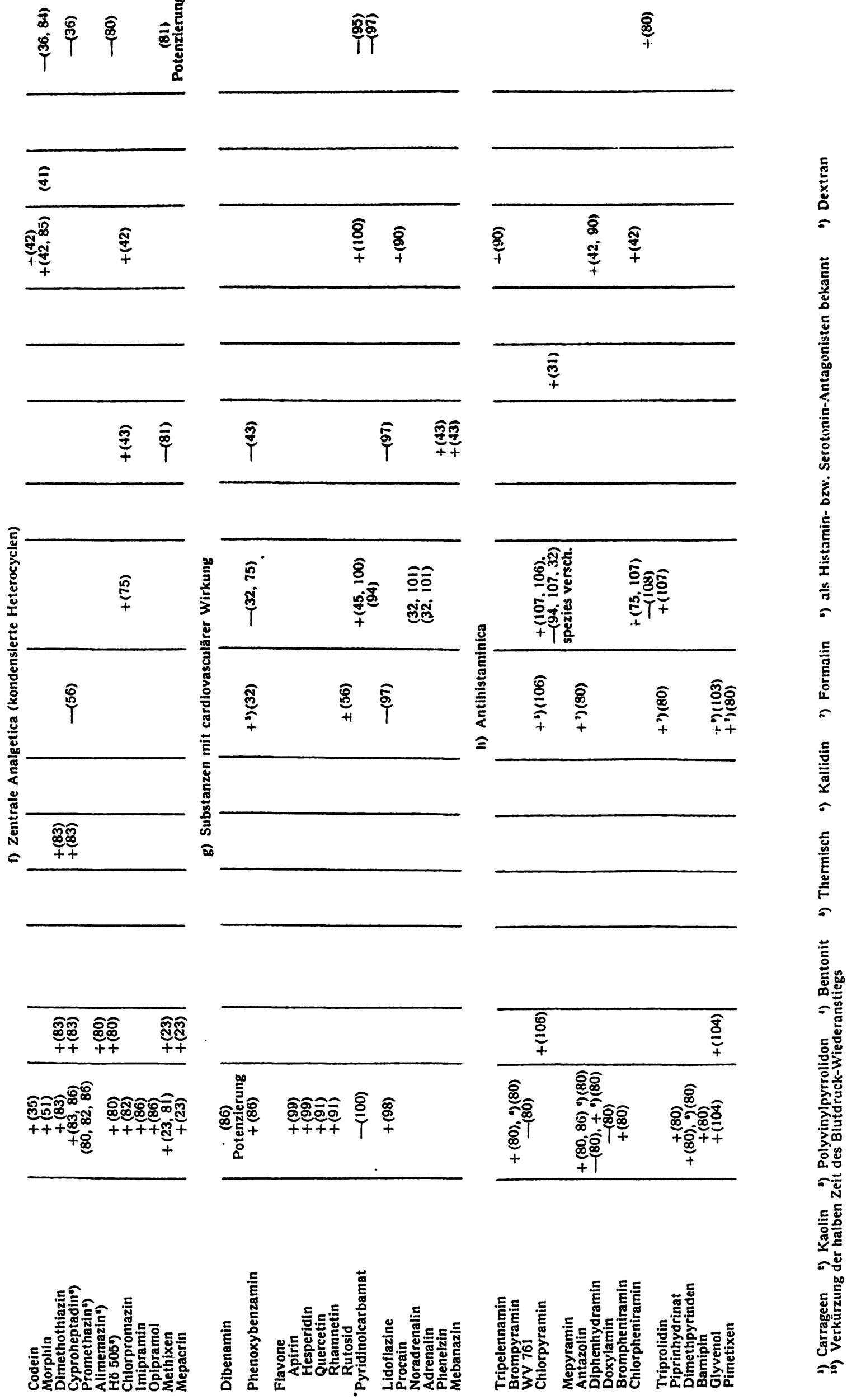

Z. klin. Chem. u. klin. Biochem. / 9. Jahrg. 1971/ Heft 2 
Thebain und die Morphin-ähnlichen Synthetica Dextrorphanol, Pethidin, Methadon und Fentanyl; ohne Wirkung waren Dextromethorphan, Cyclazocin, Pentazocin und Dextromoramid. Die Morphin-Antagonisten Malorphin und Levallorphan zeigen ebenfalls antagonistischen Effekt.

Von Rocha e Silva und Garcia-Leme $(45,86,88)$ wurde eine Reihe tricyclischer Substanzen auf ihre Kinin-antagonistische Wirkung untersucht; am isolierten Meerschweinchenileum sind Phenothiazinderivate $^{4}$ ), Iminostilbene, Dibenzcycloheptene und Thioxanthene wirksam mit gewisser Relation zur Struktur. Die meisten Substanzen hemmen nicht kompetitiv; nur in der Gruppe der Dibenzcycloheptene und Thioxanthene sind einige kompetitive Blocker $(45,88)$, was allerdings für Methixen von vaN RIEZEN (81) und für Dimethothiazin von Horowitz und MASHFord (83) rerneint wird. Alle untersuchten Substanzen hemmen reversibel, nur Dibenamin und Dibenzylen irreversibel ${ }^{4}$ ).

Im Verhältnis zu anderen Substanzklassen (z. B. den Fenamsäuren) ist eine sehr hohe Konzentration der in der Tabelle $3 \mathrm{f}$ zusammengefaßten Substanzen notwendig; nur Methixen ist beim Test am isolierten Meerschweinchenileum von besonders hoher Antikinin-Aktivität.

Andererseits hat VAN Riezen gefunden, daß Methixen als einzige bisher aus dieser Gruppe in diesem Test verwendete Substanz bei der Bradykinin-induzierten Bronchokonstriktion wirkungslos ist und die Kinininduzierte Senkung des Blutdrucks der Ratte sogar noch potenziert (81). Auch Chlorpromazin zeigt neben antagonistischer Wirkung (in vitro und in vivo) potenzierende Effekte bei einigen sehr speziellen Tests in vivo (89).

Im ganzen gesehen, ergab die lückenhafte einseitige Testung dieser Substanzgruppe (s. Tab. 3f) noch keinen deutlichen Anhaltspunkt zur Auffindung eines echten Kinin-Antagonisten; allerdings ist die Blockierung der Impulsübertragung im ZNS als gesichert anzusehen (26).

\section{Substanzen mit Hauptwirkung auf das periphere Nervensystem}

Untersuchungen über die Kinin-hemmende Wirkung von Atropin, Diphemanil-methylsulfat, Dicycloverin, Papaverin und Amphetamin sind noch sehr lückenhaft, da sie meist nur zum Vergleich mit anderen Substanzen von einer Arbeitsgruppe in eine Versuchsreihe einbezogen worden sind.

In vitro hemmt Dicycloverin die Kontraktion des isolierten Meerschweinchenileums, während Atropin einen potenzierenden Effekt ausübt (86). In vivo soll nach GreefF und Rocha e Silva der Blutdruckabfall

4) Einige der untersuchten Substanzen sind als Histamin- bzw. Serotonin-Antagonisten bekannt. nach Bradykiningabe verringert werden (58). Atropin dämpft Kinin-induzierte Schmerzwirkung nicht (90), während Papaverin und Amphetamin hier als Blocker wirken $(26,90)$. Hervol uheben ist dabei die Wirkung von Papaverin: es verhindert die Vokalisation der Versuchstiere nur, wenn Bradykinin in die A. mesenterica injiziert wird, 'nicht dagegen bei i. v. Zufuhr (90). Dieser Befund läßt den Schluß zu, daß intestinale Spasmen, von denen angenommen wird, daß sie u.a. durch Kinine verursacht werden, die Vokalisation auslösen können.

\section{Substanzen mit cardiovasculärer Wirkung (Tab. $3 \mathrm{~g}$ )}

Das Antihypertonicum Phenoxybenzamin wurde zuerst von Rocha e Silva (86) am isolierten Meerschweinchenileum getestet: in niederer Konzentration wirkt es potenzierend, in hoher Konzentration als irreversibler Bradykinin-Antagonist.

Nach Starr und West (32) bewirkt Phenoxybenzamin eine signifikante Dämpfung des thermischen Ödems, was die Autoren damit erklären, daß die $\alpha$-adrenergischen Rezeptoren blockiert werden, so daß mehr freizirkulierende Catecholamine die Bradykininwirkung $\mathrm{zu}$ reduzieren vermögen.

Flavone erwiesen sich als Bradykinin-Antagonisten beim Test am Meerschweinchenileum. Nachdem Quercetin ein stärkerer Antagonist als Rhamnetin ist, sind offensichtlich die Positionen 5 und 7 der Hydroxylgruppen für den Hemmeffekt von Bedeutung (91).

Nach orientierenden Versuchen am Menschen unterbleibt bei Guanethidinprämedikation die Venokonstriktion (am Unterarm) nach Bradykiningabe, die Venodilatation ist unverändert. Der arterielle Blutdruckabfall bleibt ebenfalls unbeeinflußt.

Das von Shimamoto verwendete Pyridinolcarbamat ist als einziger spezifischer Blocker der Kinin-induzierten Venokonstriktion in vivo und in vitro bekanntgeworden $(92,93) .50-400 \mu \mathrm{g} / \mathrm{m} l$ hemmen die durch Bradykinin hervorgerufene Kontraktion isolierter venöser Gefäße vom Kaninchen (100). Dagegen konnte Sнimamoto (100) keinen hemmenden Einfluß auf die Bradykininwirkung an der isoliert perfundierten Meerschweinchenlunge, am isolierten Meerschweinchenileum und am isolierten Rattenuterus feststellen. Dieser Befund wurde für den Rattenuterus von WERLE (95) bestätigt. Die Hemmung durch Pyridinolcarbamat ist als kompetitiv und reversibel beschrieben. Die Substanz verhindert in vivo das Auftreten der passiven cutanen Anaphylaxie und die Ödementstehung nach mechanischen und thermischen Traumen. Von Willoughry und Mitarbeitern (94) wurden die Versuche von SHIMAMOTO hinsichtlich der Hemmung der Permeabilitätserhöhung durch Bradykinin (und andere Permeabilitätsfaktoren) bestätigt. Nach den Untersuchungen von ZWWerFACH (75) wirken Kinine an den Gefäßen der Endstrombahn nur dilatierend. Das würde bedeuten, daß die Vasokonstriktion kein Kinineffekt ist und die antagonistische Wirkung von Pyridinolcarbamat sich 
auf andere Wirkungsmechanismen beziehen müßte. Auch soll nach Sнmмamoto eine Rekanalisierung obsturierter Gefäße erfolgen (96), ein Vorgang, der durch die Ausschaltung des Kinin-Systems begünstigt wird. Lidoflazine (1-[4,4-Di-(fluorophenyl)-butyl]-4-[2,6-dimethyl. (aminocarbonyl)-methyl]-piperazin) wurde zunächst beschrieben als langwirkende Coronargefäß-crweiternde Substanz, hat daneben antagonistische Wirkung gegenüber Angiotensin, Serotonin und Histamin (Lit. in 97). Auf seine Bradykinin-antagonistische Wirkung wurde es in vitro und in vivo von TürkER und Mitarbeitern (97) und JANSEN (98) geprüft; bis auf die Blockierung der Kinin-induzierten Ileumkontraktion waren alle Befunde negativ (s. Tab. $3 \mathrm{~g}$ ).

\section{Histamin- und Serotonin-Antagonisten (Tab. 3h)}

Nach Collier und Mitarbeitern (54), die die Gruppe der Analgetica und Antipyretica besonders in Hinsicht des Effektes bei der Bronchokonstriktion gründlich bearbeiteten, sollen Antagonisten von Histamin und Serotonin die Kinin-induzierte Bronchokonstriktion nicht blockieren. So sind Beobachtungen von BERNAUER und Mitarbeitern (102) über die Reduzierung des Bronchospasmus im anaphylaktischen Schock (z. B. durch Mepyramin) auf den Histamin-Antagonismus zurückzuführen.

Doch sind einige Substanzen mit Hemmeffekt gegenüber anderen, speziellen Kininwirkungen aufgefunden worden. Es gehören dazu Äthylendiaminderivate, Äthanolaminderivate, Propylaminderivate u. a. und Glyvenol.

Am isolierten Meerschweinchenileum ist die Hemmung der Bradykinin- und Kallidinwirkung praktisch gleich stark (80). Die Substanzen mit höchster AntikininAktivität am isolierten Ileum sind Dimethpyrinden und Hö-505. Offensichtlich sind nach Befunden von WERLE und Lorenz (80) die Rezeptoren des Meerschweinchenileums für Kinine und Acetylcholin sehr ähnlich. Dagegen ist die Hemmung der Kallidinwirkung auf den isolierten Rattenuterus bei allen getesteten Substanzen mit $1 / 3$ der zur Bradykininhemmung notwendigen Dosierung erreichbar.

Von allen hier besprochenen Substanzen ist Dimethpyrinden die einzige, die die Kinin-induzierte Blutdrucksenkung am Hund vermindert, nicht nur die Zeit des Wiederanstiegs verkürzt. Diese Beobachtung wurde mit Bradykinin, Kallidin (Lys-Bradykinin) und Met-Lys-Bradykinin in gleicher Weise gemacht (80).

Die Antikinin-Aktivität dieser Substanzen tritt bei den verschiedenen Tests so unregelmäßig auf, daß auch aus dieser Substanzgruppe kein Kinin-Antagonist im engeren Sinn hervortritt.

Glyvenol (Äthyl-3,5,6-tri-O-benzyl-D-glucofuranosid) wurde von JAQUES, SCHÄR und Mitarbeitern synthetisiert und auf seine antagonistische Wirkung getestet (103, 104). An isolierten Organen erwies es sich als Antagonist gegenüber Histamin, Serotonin, Acetylcholin, Vasopressin, Angiotensin, Wespengift, slow reacting substance, Trypsin und nicht zuletzt Bradykinin (s. Tab. $3 \mathrm{~g}$ ).

Pathologische Vorgänge, an denen das Kinin-System beteiligt ist, werden blockiert, so Antigen-AntikörperReaktion, Endotoxin-induzicrte I eukocytenmigration, Schock, Arthusphänomen (103). In hoher Dosierung wird das Dextranödem an der Rattenpfote gehemmt. Die direkte Kininbeeinflussung wurde allerdings in vivo nicht geprüft.

Die Untersuchungen über den Kinin-Antagonismus der Antihistaminica sind noch in den Anfängen. So können orientierende Versuche von AMIUNDSEN und Mitarbeitern $(59,105)$ über Histaminfreisetzung durch Kallikrein zusammen mit Phospholipase A nur spekulativ herangezogen werden zu einer Deutung des Mechanismus dahingehend, daß Antihistaminica über eine Histaminblockierung eine Kininblockierung vortäuschen.

\section{Kritische Betrachtungen}

zum Wirkungsmechanismus der Kininblockierung

Nach dem Stand der Forschung kann ausgeschlossen werden, daß die besprochenen Substanzgruppen in den Mechanismus der Kininliberierung eingreifen; allerdings stehen Untersuchungen über die Beeinflussung des dem System vorgeschalteten Reaktionsmechanismus noch aus (z. B. Hageman-Faktor oder PräkallikreinAktivierung).

Die mit synthetischen Kininen durchgeführten Experimente sind zwar zahlreich, aber es muß deutlich gesagt werden, daß manche Arbeitsgruppen die Geltungsbereiche ihrer Experimente nicht schildern, keine weiteren pharmakologischen Teste ausführen (vielleicht negative Befunde nicht angeben) und damit dem mit dem Gebiet nicht vertrauten Leser die in einer Arbeit referierten Ergebnisse oft klar erscheinen müssen.

So ist ein kritischer Punkt, daß die meisten Arbeitsgruppen viele Substanzen an einem ihnen geläufigen Kinin-Testobjekt prüfen und den einen speziellen positiven Befund verallgemeinern, wobei die Brauchbarkeit dieses einen Tests manchmal noch in Frage gestellt ist. Dazu tritt auch meist der Effekt ein, daß geringe Dosis-Verschiebungen auch eine Verschiebung von Hemmung zu Potenzierung oder eine Aufhebung des Effektes zur Folge haben können.

So schreibt Bonta (55): „,even today, following many years of successfull experience with such preparations in human therapy, the pharmacologists are still trying to find the best correlating experimental models... During a study of recognized anti-inflammatory drugs, even in fairly simple experimental designs, the pharmacologist is still frequently faced with puzzling questions."

Eine der größten Schwierigkeiten ist die Auswahl der Spezies für die Versuche, was an einem Beispiel erläutert sei:

Die Antihistaminica Mepyramin und Triprolidin, hemmen die Bradykinin- wie Histamin-induzierte Er- 
höhung der Kapillarpermeabilität bei Ratten und Mäusen; bei Kaninchen ist der Hemmeffekt gegenüber Bradykinin wesentlich herabgesetzt und bei Meerschweinchen ist er kaum zu beobachten (107). Dieser letztere Befund ist bereits seit 1957 bekannt (109).

Auch beim Menschen werden Quaddelbildung und Erythem, die durch lokale Bradykinin-Injektion ausgelöst werden, durch Antihistaminica nicht gehemmt (110).

Diese Speziesunterschiede unterstützen die Theorie von ZweIfaCH, daß einerseits die Permeabilitätserhöhung durch Kinine mit einer Histaminfreisetzung verknüpft sein kann, andererseits ein direkter Kinineffekt vorliegt (75). Derartige vergleichende Untersuchungen bei verschiedenen Spezies, die nur von den wenigsten Arbeitsgruppen vorgenommen worden sind, sind Hinweise auf die einleitend bereits angedeuteten Lücken und die Forderung nach systematischer Bearbeitung.

Am Beispiel des Rattenpfotenödems sei ein anderer kritischer Punkt diskutiert: als Modell der Entzündung hält es hinsichtlich eines Testes auf Kininbeteiligung nur eingeschränkt stand (56); außerdem spielt das Alter der Versuchstiere in diesem Experiment (wie auch bei der Vokalisation der Ausdruck des Schmerzes) eine nicht unbedeutende Rolle (66, 90). Nach AARSEN (111) scheint es erwiesen, daß Bradykinin in niedrigster Konzentration die Permeation von Histamin beschleunigt, ein Befund, der bei den unterschiedlichen Ergebnissen mit experimentellen Ödemen vielleicht in Betracht gezogen werden muß.

Die (bei vielen Substanzgruppen noch nicht vorgenommene) Prüfung der Blockierung der hypotensiven Kininwirkung hat die einzige abweichende Reaktion erbracht: Nur Dimethpyrinden vermindert die Senkung des arteriellen Blutdrucks des Hundes, alle anderen geprüften Substanzen bewirken eine Verkürzung der hypotensiven Phase, d. h. der Wiederanstiegszeit auf $50 \%$ der Druckdifferenz. (Der von Lecomte $(62,112)$ für Phenylbutazon erhobene Befund konnte von keiner Arbeitsgruppe bestätigt werden.) Dazu ist allerdings zu bemerken, daß nach VARGAFtrg (38) die durch Kininogenasen ausgelöste Blutdrucksenkung in eben derselben Weise beeinflußt wird. Von anderen Reaktionsmechanismen übertragen, könnte dieser Befund bedeuten, $\mathrm{da}$ ein um den Rezeptor konkurrierender Blocker langsamer eingreift als die Stimulierung des Rezeptors durch Bradykinin erfolgt.

Nachdem bisher die Suche nach dem Kinin-Antagonisten (mit Ausnahme von Phenylbutazon) erfolglos geblieben ist, haben die Experimente der verschiedensten Arbeitsgruppen doch einige Hinweise auf die Eigenschaften der Kinin-Rezeptoren erbracht.

$\mathrm{Zu}$ diskutieren wäre auch eine Veränderung im Wechselspiel Kinine-Catecholamine in dem Sinn, daß die Gegenregulation durch erhöhten Catecholaminspiegel rascher erfolgt.

Botha (113) nimmt an, daß um die Kapillaren ein zuführendes System nicht-myelinhaltiger, freiverzweigter, paravasculärer Rezeptoren liegt. $\mathrm{Da}$ die Ansprechbarkeit dieser Rezeptoren je nach dem Grad der Stimulierung variieren kann, fordern BотнA und andere Autoren zur Austestung ansteigende Dosierung des Stimulans gegen eine konstante Dosis des Antagonisten $(85,114)$.

Collier nimmt an, daß die Antagonisten wirksam werden durch die Blockade eíhes Weges der Kinine zu oder von den spezifischen Rezeptoren und nicht durch die Blockierung der Rezeptoren selbst (17). Die Blockade kann sich z. B. auf zelluläre SchrankenPassage beziehen oder auf Freisetzung von Intermediärsubstanzen (115). Diese Theorie wird $z$. B. durch den Befund unterstützt, daß die Bronchokonstriktion nur blockiert wird, wenn der Antagonist zeitlich vor dem Kinin verabreicht wird, was bedeutet, daß nicht die letztlich erfolgende Kontraktion des Muskels gehemmt wird.

Schließlich ist nicht damit zu rechnen, daß die Rezeptoren der verschiedenen Erfolgsorgane gleich gebaut, also gleichsinnig inhibiert werden können. Damit könnten positive/negative Befunde gedeutet werden, d. h. die Ergebnisse, die nur mit einem einzigen Modell erzielt werden, können nicht verallgemeinert werden.

Nach Guzman (64) ist der ruhende Schmerz-Rezeptor chemo-sensitiv. Schmerz wird ausgelöst durch verschiedenartige Faktoren, z. B. Kationen, Amine, Peptide. Daraus zieht Guzman den Schluß, daß die Schmerzrezeptoren der Peripherie nicht struktur-, sondern ladungsspezifisch sind. Die Entzündungshemmung durch Acetylsalicylsäure und Indomethacin wird durch gleichzeitige Applikation von Cystein abgeschwächt (116). Dieser, nicht im Zusammenhang mit Untersuchungen auf dem Kiningebiet erhobene Befund deutet auf ihre Wirkung auf Sulfhydrylgruppen, da ein SHDonator mit ihnen in Konkurrenz treten kann.

Kinin-Injektion in eine Mesenterialarterie löst eine 15-30 Sek. dauernde Schmerzreaktion aus $(117,118)$. Diese Erscheinung kann unterdrückt werden durch alle bekanñten nicht-narkotischen Analgetica und GuzMaN konnte in interessanten Experimenten differenzieren zwischen einer Blockierung in der Peripherie und im Zentralnervensystem. In Versuchen mit gekreuzter Milzperfusion vermögen periphere Analgetica, einem Spenderhund appliziert, Schmerzreaktion eines Empfängerhundes $\mathrm{zu}$ unterdrücken. Dagegen ist Morphin beim Empfängertier wirkungslos, wenn es dem Spenderhund appliziert wird. Nur Applikation beim Empfängertier direkt verhindert dessen Schmerzreaktion (64). Hier liegt also eine Blockade im ZNS vor.

Im allgemeinen hat sich die Ansicht durchgesetzt, daß bei der Beurteilung der Wirkung der behandelten Substanzen die Beeinflussung der Kininwirkung nicht mehr außer Acht gelassen werden darf. Von einigen Arbeitsgruppen (z.B. 19, 56) ist die Forderung gestellt worden, in den screening test entzündungshemmender Substanzen die Antikininwirkung mit einzubeziehen und zwar in vitro und in vivo. 


\title{
Buddecke
}

\section{Grundriß der Biochemie}

\section{für Studierende der Medizin, Zahnmedizin und Naturwissenschaften}

\author{
Von Prof. Dr. Eckhardt Buddecke \\ 2. Auflage \\ Mit mehr als 400 Formeln, Tabellen und Diagrammen \\ XXXII, 499 Seiten. 1971. Plastik flexibel DM 27,50
}

Stoffe und Stoffwechsel

Chemische Zusammensetzung und Stoffwechsel der Zelle - Der Stoffwechsel als chemische Reaktionsfolge Enzyme - Coenzyme - Aminosäuren - Nucleinsäuren Proteine - Glucose und Kohlenhydrate - Lipide - Citratzyklus und biologische Oxydation - Porphyrine - Wasserghaushalt - Mineralhaushalt

Stoffwechselregulation

Selbstregulation durch Rückkopplung - Regulation durch Metabolitkonzentrationen - Enzymkonkurrenz Hormone

\section{Inhaltsübersicht}

\section{Helmut A. Fischer - Gottfried Werner Autoradiographie}

Oktav. IX, 214 Seiten. Mit 93 Abbildungen und 14 Tabellen. 1971. Werkstoff DM 42,-

In den letzten Jahren hat die Methodik der Autoradiographie in zahlreichen naturwissenschaftlichen Forschungsgebieten Anwendung gefunden. In dem vorliegenden Buche „Autoradiographie“ werden - nach einer kurz gehaltenen Einleitung über die allgemeinen physikalischen Grundlagen - erstmals alle wesentlichen Techniken für die verschiedenen Anwendungsgebiete beschrieben, typische Beispiele aus den betreffenden Fachgebieten (z. B. Biologie, alle Gebiete der Medizin, Chemie, Physik, Biochemie, Kriminalistik, Pharmakologie und Toxikologie) gegeben und die Möglichkeiten und Grenzen der qualitativen und quantitativen Autoradiographie kritisch behandelt. Die Gliederung des Stoffes nach der Dimension (makroskopisch, mikroskopisch und elektronenmikroskopisch) der autoradiographisch untersuchten Objekte ergibt eine gute Übersichtlichkeit der sehr differenten Anwendungsgebiete. Die, relativ zur verarbeiteten Gesamtliteratur von ca. 10000 Literaturzitaten, geringe Zahl von 500 textbezogenen Literaturangaben soll das Auffinden der in den verschiedenen Zeitschriften weit gestreuten Publikationen erleichtern.

\section{Walter de Gruyter · Berlin · New York}




\title{
ACTA BIOCHIMICA ET BIOPHYSICA \\ ACADEMIAE SCIENTIARUM HUNGARICAE
}

\author{
(III) \\ Zeitschrift der Ungarischen Akademie der Wissenschaften,
}

VOLUME 5

NUMBER 3

Contents

A. RaAfat, Judithstur, M. Sipos, N. Marek: Some Aspects of Oxidative-Reductive Changes in Chloroplast Suspensions During the Process of Ageing

J. Sümegr, S. Damjanovich, F. Szeszák, A. Daróczy: The Effect of X-rays on the Salt Stimulation of DNADependent RNA Synthesis in Vitro

S. Danjanovich, S. Tótr, J. Sümegr: Studies on the Fluorescence of Glycogene Phosphorylases

A. Zsindeț, J. Aradr, B. Tankó: On the Mechanism of the Degradation of Nucleic Acids by Periodate Oxidation

G. LÉNÁRT, I. ÁRKY, G. RISCHÁK: Determination of the ${ }^{85} \mathrm{SR}^{++} / \mathrm{Ca}^{++}$Ratio in Bone (Short Communication)

L. Gráf, G. CSeh, I. NAgY, M. Kurcz: An Evidence for Deterdation of Prolactin Monomer (Preliminary Communication)

L. Gráf, E. Barát, G. Csem, M. Sajgó: Amino Acid Sequence of Porcine $\gamma$-Lipotropic Hormone (Preliminary Communication)

Ilma SzÁsz, G. GÁrdos: The Effect of Urea and Related Compounds on the Morphology and Energy Metabolism of Erythrocytes (Preliminary Communication)

Széphalmi, G.: A Stochastic Model for Stimulus-Irritation Processes

G. MAsszi: The Dielectric Characteristics of Bound Water

E. Egyed, J. Trgyr: The Influence of $\beta$-Radiation of Tritium on the Excitation and Ion Content of the Striated Muscle

L. NAGY: Semiconductor Property of Frog Sartorius Muscle

R. A. Chaplarn, E. Pfister: On the Application of the Phenomenological Treatment of Irreversible Thermodynamics to Contracting Muscle

L. Homola: Water Circulation Caused by Hindered Diffusion and Unequal Distribution of Solutes

Book Reviews

ACTA BIOCHIMICA ET BIOPHYSICA erscheint vierteljährlich in Heften $z u$ einem Band von etwa $400-500$ Seiten. Format: $17 \times 25 \mathrm{~cm}$.

Abonnementspreis pro Band: $\$ 16.00 ; \mathrm{DM} 64,-$

Vertrieb: KULTURA, Budapest 62, Postfach 149; Auslieferung für das Gebiet der Deutschen Bundesrepublik: KUNST UND WISSEN, Erich Bieber, Stuttgart S., Wilhelmstraße 4

AKADÉMIAI KIADO, Verlag der Ungarischen Akademie der Wissenschaften, Budapest 502. Postfach 24 


\section{Literatur}

1. Schröder, E. und R. Hemper, Experientia, Basel 20, 529 (1964). - 2. Schröder, E. und K. LüBke, The Peptides, New York (1966). - 3. Frey, E. K., H. KraUt, E. Werle, R. Vogel, G. ZICKGRAF-RÜDEL und I. Trautschold, Das KallikreinKinin-System und seine Inhibitoren, Stuttgart (1968). - 4. Stewart, J. M., Federation Proc. 27, 63 (1968). - 5. Erdös, E. G., Biochem. Pharmacol. 8, 112 (1961). - 6. Stewart, J. M. und D. W. Woolley, in E. G. ERDös, N. BACK und F. SrCuteri, Hypotensive Peptides, New York (1966). - 7. Strwart, J. M. und D. W. WoolLeY, Nature 206, 619 (1965). - 8. STEWART, J. M., Federation Proc. 26, 466 (1967). - 9. Vogler, K. und P. LANz, in E. G. ERdös, N. BACK und F. Sicutrri, Hypotensive Peptides, New York (1966). - 10. Yamamura, Y., Tanabe Amino Acid Research Foundation, Annual Report, Osaka (1968). - 11. Coliter, H. O. J., J. A. Holgate, M. Schachter und P. G. SHORLEY, Brit. J. Pharmacol. 15, 290 (1960). - 12. Collier, H. O. J. und P. G. SHORLEY, Brit. J. Pharmacol. 15, 601 (1960). 13. BhoolA, K. D., H. O. J. Collier, M. Schachter und P. G. Shorley, Brit. J. Pharmacol. 19, 190 (1962). - 14. Collier, H. O. J., Ann. N. Y. Acad. Sci. 104, 290 (1963). - 15. G juruš, V. und E. WestermanN, Nature, London 205, 402 (1965). - 16. Colrrer, H. O. J., Biochem. Pharmacol. 10, 47 (1962). - 17. Collier, H. O. J., G. W. L. JAMES und C. Schinemder, Nature, London 212, 411 (1966). - 18. AArsen, P. N., Brit. J. Pharmacol. 27, 196 (1966). - 19. Gryglewski, R. J., B. Panczenko, Z. Gorka, A. Chyrkowski und A. ZMudA, Dissert. Pharm. Pharmacol. 21, 1 (1969). - 20. Moog, D. und K. GreEFF, NaunynSchmiedeberg's Arch. exp. Path. Pharmakol. 246, 16 (1963). 21. AArsen, P. N., Acta physiol. pharmacol. Neerl. 14, 1 (1966). 22. Davies, G. E., G. Holman, T. P. Johnston und J. S. Lowe, Brit. J. Pharmacol. 28, 212 (1966). - 23. Huidrobro, H. V. und H. A. GonzÁles, Abstracts III. Pharmacol. Congr., Symp. on Vasoactive Peptides, Sao Paulo 1966. - 24. LykkE, A. W. J. D. A. Wirloughry und E. R. KosChe, J. Path. Bact. 94, 381 (1967). - 25. Martelli, E. A., J. Pharm. Pharmacol. 19, 617 (1967). - 26. LIM, R. K. S., F. GuzMaN, D. W. RODGERS, K. Goto, C. Braun, C. D. Dickerson und R. J. Engle, Atch. internat. pharmacodyn. thérap. 152, 25 (1964). - 27. Coffman, J. D., Clin. Pharmacol. Therap. 7, 26 (1966). - 28. Collier, H. O. J., Proc. 1st Int. Pharmacol. Meeting, Stockholm 1961, Vol. 9, S. 47, Oxford 1963. - 29. GJuRIš, V., B. Heicke und E. WestermanN, Naunyn-Schmiedeberg's Arch. exp. Pathol. Pharmakol. 247, 429 (1964). - 30. GJuriš, V., B. Heicke und E. Westermann, Naunyn-Schmiedeberg's Arch. exp. Pathol. Pharmakol. 248, 540 (1964). - 31. StresemaNN, E., Acta allerg., K'hrn. 18, 235 (1963). - 32. Starr, M. S. und G. B. West, Brit. J. Pharmacol. 31, 178 (1967). - 33. Adams, S. S., K. F. MCCuLLovgr und J. S. Nicholson, Arch. internat. pharmacodyn. thérap. 178, 115 (1969). - 34. WINDER, C. V., J. WAX und M. WelFord, J. Pharmacol. exp. Therap. 148, 422 (1965). - 35. Colirer, H. O. J. und P. G. Shordex, Brit. J. Pharmacol. 20, 345 (1963). - 36. Vargaftig, B., Experientia, Basel 22, 182 (1966). - 37. TÜrRER, K. und B. K. KIRAN, Arzneimittel-Forsch., Aulendorf 14, 1318 (1964). - 38. Vargaftig, B. B., Med. Pharmacol. exp. 17, 517 (1967). - 39. Collier, H. O. J., L. C. Dinneen, A. C. Perkins und P. J. Piper, Naunyn-Schmiedeberg's Arch. exp. Path. Pharmakol. 259, 159 (1968). - 40. SicuterI, F., G. Franchi, M. Fanciullacci, P. L. Del Bianco und A. BAvazzano, Pharmacol. Res. Commun. 1, 195 (1969):- 41. KrautHAMER, G., R. K. S. Lim, F. GuzMan und R. R. Fulp, Pharmacol. Res. Commun. 1, 170 (1969). - 42. Burns, R. B. P., N. J. Alioto und K. E. HurLey, Arch. internat. Pharmacodyn. thérap. 175, 41 (1968). - 43. Collier, H. O. J., G. W. L. James und P. J. Piper, Brit. J. Pharmacol. 34, 76 (1968). - 44. Collier, H. O. J. und G. W. L. James, Brit. J. Pharmacol. 30, 281 (1967). - 45. GArcia Leme, J. und M. Rocka e Sizva, Brit. J. Pharmacol. 25, 50 (1965). - 46. van RuEzen, H. und E. Betrink, J. Pharm. Pharmacol. 20, 474 (1968). - 47. WinTER, C. A., Int. Symp. on Nonsteroidal Anti-infiammatory Drugs, Milan 1964, Exc. Med. Found. Amsterdam (1965). - 48. WINTER, C. A. und L. FLATAKER,
J. Pharmacol. exp. Therap. 148, 373 (1965). - 49. BAuER, G., R. GMEEINER und H. KonzEtT, Naunyn-Schmiedeberg's Arch. exp. Pathol. Pharmakol. 251, 182 (1965). - 50. Vargaftig, B. und M. Born, Thérapie, Paris 24, 513 (1969). - 51. Berrr; P. A. und H. O. J. Collier, Brit. J. Pharmacol. 23, 201 (1964). 52. Collier, H. O. J., Ann. N. Y. Acad. Sci. 104, 290 (1963). 53. Back, N., M. Jainchill, H. J. Wilkens und J. L. Ambrus, Med. pharmacol. exp. 15, 597 (1966). - 54. Collier, H. O. J., J. A. Holgate, M. Schachter und P. G. Shorley, J. Physiol. 149, 54 P (1959). - 55. BontA, I. L. und C. J. DE Vos, European J. Pharmacol. 1, 222 (1967). - 56. BontA, I. L., Acta physiol. pharmacol. Neerl. 15, 188 (1969). - 57. Vargaftig, B. B., N. Bhargava, C. J. de Vos und I. L. Bonta, Pharmacol. Res. Commun. 1, 203 (1969). - 58. Graeff, F. G., I. R. Pela und M. Rocha e Silva, Pharmacol. Res. Commun. 1, 157 (1969). 59. AMundsen, E., E. Ofstad und P.-O. HAGEN, Arch. internat. pharmacodyn. thérap. 178, 104 (1969). - 60. Lecomte, J. und J. Troquet, Arch. Intern. Physiol. 70, 735 (1962). - 61. YAMAmoto, H., C. Saito, T. Okamoto, H. Awata, T. InUkai, A. Hrrohashi und Y. Yukawa, Arzneimittel-Forsch., Aulendorf 19, 981 (1969). - 62. Lecomte, J. und J. Troquet, Compt. rend. Soc. Biol., Paris 154, 1115 (1960). - 63. MarEK, J., 4th International Congress on Pharmacology, Bascl (1969). - 64. GuzMav, F. und R. K. S. Lim, Arch. Biol. Med. Exper. 4, 180 (1967). 65. Grimovi, J. und Z. HorAkovi, 4th International Congress on Pharmacology, Basel (196\%). - 66. Giordano, F. und U. ScapagninI, Med. Pharmacol. exper. 17, 445 (1967). - 67. Newbould, B. B., Brit. J. Pharmacol. 35, 487 (1969). - 68. Lurie, M. B., Ann. N. Y. Acad. Sci. 52, 1074 (1950). - 69. BontA, I. L. und C. J. DE Vos, Acta endocr., K'hvn 49, 403 (1965). 70. Shimamoto, T. und T. Ishioka, Proc. Japan. Acad. 43, 342 (1967). - 71. Ishioka, T., Y. Honda, A. Sagara und T. Shimaмoro, Acta endocr., K'hvn. 60, 177 (1969). - 72. Cline, M. J. und K. L. Melmon, III. Pharmacol. Congress, Symp. on Vasoactive Polypeptides Sao Paulo (1966). - 73. EISEN, V., L. GreenBaum und G. P. Lewrs, Brit. J. Pharmacol. 34, 169 (1968). 74. Cline, M. J. und K. L. Melmon, Science, Washington 153, 1135 (1966). - 75. ZWeIFACH, B. W., in E. G. ERDös, N. BACK und F. Sicuteri, Hypotensive Peptides, New York (1966). 76. Frank, L., Y. Rapp, L. Biro und F. S. Glickman, Arch. Dermat. Syph., Chicago 89, 115 (1964). - 77. JEzdinsKx́, J., P. OChonskY, V. JEZDinska und J. Marek, 4th International Congress on Pharmacology, Basel (1969). - 78. Lewis, G. P. und E. RerT, Brit. J. Pharmacol. 26, 444 (1966). - 79. TreNDelenburg, U., J. Pharmacol. Exp. Therap., Baltimore 154, 418 (1966). - 80. WERLE, E. und W. LORENZ, Vortrag Int. Symposium Fiesole 1969, in F. Sicuteri, M. Rocha E Silva und N. BaCK, Bradykinin and Related Kinins, S. 447, New York (1970). 81. van Riezen, H., J. Pharm. Pharmac. 18, 688 (1966). - 82. Mariani, L., Boll. Soc. ital. biol. sper. 37, 1481 (1961). - 83. Horowitz, J. D. und M. L. MAshrord, J. Pharmacy Pharmacol., London 21, 51 (1969). - 84. Pearson, L. und W. J. Lang, Europ. J. Pharmacol. 6, 17 (1969). - 85. BothA, D., F. O. Müller, F. G. M. KRUeger, H. Melnitzky, L. VermaAk und L. Louw, Europ. J. Pharmacol. 6, 312 (1969). - 86. Rocha E Silva, M. und J. Garcia Lenre, Med. exp. 8, 287 (1963). 87. Konzetr, H. und H. Bauer, Z. Physiol. Chem. 349, 927 (1968). - 88. Rocifa E Silva, M. und J. Garcia Leme, NaunynSchmiedeberg's Arch. exp. Pathol. Pharmakol. 250, 167 (1965). 89. Krivoy, W., D. Kroeger, B. Moberley und R. Porter, III. Int. Pharmacol. Congress, Symp. on Vasoactive Polypeptides, Sao Paulo (1966). - 90. Taira, N., K. Nakayama und K. HashiMoto, Tohoku J. Expcr. Med. 96, 365 (1968). - 91. ChaU, T. T. und T. J. HALEY, J. pharm. Sci. 58, 621 (1969). 92. Shimamoto, T. und H. Maszawa, III. Int. Pharmacol. Congress, Symp. on Vasoactive Polypeptides, Sao Paulo (1966). - 93. Shimamoto, T., F. Numano, T. Fujita, T. Ishroka und T. Arsumr in E. G. Endös, N. BACK und F. Srcuterr, Hypotensive Peptides, New York (1966). - 94. LYKKE, A. W. J., D. A. WILlOUghbY und E. R. KosChe, J. 
Path. Bact. 97, 527 (1969). - 95. WERLE, E., Vortrag gehalten bei Geigy A.G. Pharma, Basel 1969. - 96. Shimamoto, T., F. Numano und T. FujITA, Amer. Heart J. 71, 216 (1966). - 97. TürkER, R. K., Ș. Kaymakçalan, S. Kayan und G. Çetin, Europ. J. Pharmacol. 4, 175 (1968). - 98. Janssen, P. A., Personal communication in 1. c. (97). - 99. Garcia Leme, J. und E. J. WaLASZEK, Pharmacologist 9, 242 (1967). - 100. ShIMAMOTo, T., H. Maezawa, H. Yamazaki, T. Atsumi, T. Fujita, T. Ishioka und T. Sunaga, Amer. Heart J. 71, 297 (1966). - 101. Brown, R. A. und G. B. West, J. Pharmacy Pharmacol. London 17, 119 (1965). - 102. Bernauer, W., F. Hahin und H. Giertz, Arch. internat. Pharmacodyn. thérap. 178, 137 (1969). - 103. Jaques, R., G. Huber, L. Neipp, A. Rossi, B. Schïr und R. MeIER, Experientia, Basel 23, 149 (1967). - 104. JAQUES, R. und B. Schär, Schweiz. med. Wschr. 97, 553 (1967). - 105. Amundsen, E., E. Ofstad und P. O. HAGEN, Pharmacol. Res. Commun. 1, 130 (1969). - 106. GeCSE, A., L. Szekeres und G. B. West, J. Pharmacy Pharmacol., London 21, 544 (1969). - 107. BECKER, E. L., I. MotA und D. Wong, Brit. J. Pharmacol. 34, 330 (1968). -
108. JANOFf, A., Nature, London 212, 1605 (1966). - 109. Holdstock, D. J., A. P. Mathias und M. Schachter, Brit. J. Pharmacol. 12, 149 (1957). - 110. Greaves, M. und S. Shuster, J. Physiol. 193, 255 (1967). - 111. AArsen, P. N., Acta physiol. pharmacol. Neerl. 15, 223 (1969). - 112. Lecomte, J. und J. Troquet, Compt. rend. Soc. Biol., Paris 158, 211 (1964). - 113. Botha, D., F. O. MÜLleR, F. G. M. KRUEGer, H. MelnitzKY, L. VermaAK und L. Louw, 4th International Congress on Pharmacology, Basel (1969). - 114. Irm, R. K. S., D. G. MILLER, F. Guzman, D. W. Rodgers, R. W. Rodgers, S. K. WaNG, P. Y. ChaO und T. Y. ShrH, Clin. Pharmacol. Therap. 8, 521 (1967). - 115. Collier; H. O. J., Pharmacol. Res. Commun. 1, 146 (1969). - 116. OronsKy, A. L., L. Triner, O. S. SteINSLAND und G. G. NaHAS, Nature, London 223, 619 (1969). - 117. Braun, C., F. Guzman, E. W. Horton, R. K. S. Lim und G. D. Potrer, J. Physiol. 155, 13'P (1961). - 118. Guzman, F., C. Braun, R. K. S. Lm, G. D. Potrer und D. W. Rodgers, Arch. internat. pharmacodyn. thérap. 149, 571 (1964).
Dr. Rosmarie Vogel 8000 München 15 Nussbaumstr. 20 